\title{
THE BASIC POWER SERIES OF INTERPOLATION*
}

BY

GEORGE RUTLEDGE

1. Introduction. The coefficients of the polynomial determined by the $2 n+1$ points

$$
\left(-n h, y_{-n}\right), \cdots,\left(-h, y_{-1}\right),\left(0, y_{0}\right),\left(h, y_{1}\right), \cdots,\left(n h, y_{n}\right)
$$

have been studied extensively by the author in a paper $\dagger$ to be referred to hereafter as (I).

There is no essential loss of generality in placing $h=1$ and $y_{0}=0$ in (1). Then the polynomial, $P^{(2 n)}(x)$, has the form (I, (31), (22)):

$$
\begin{aligned}
& \sum_{i=1}^{n} \sum_{k=1}^{n-i+1} \sum_{j=i+k-1}^{n} j\left(\begin{array}{c}
j+i-k-1 \\
2 i-2
\end{array}\right) \frac{A_{i j}^{(n)}}{2 D^{(n)}}\left(\Delta_{-k}^{[2 i-1]}+\Delta_{k}^{[2 i-1]}\right) x^{2 i-1} \\
+ & \sum_{i=1}^{n} \sum_{j=i}^{n}\left(\begin{array}{c}
j+i-1 \\
2 i-1
\end{array}\right) \frac{A_{i j}^{(n)}}{2 D^{(n)}} \Delta_{0}^{[2 i]} x^{2 i} \\
+ & \sum_{i=1}^{n} \sum_{k=1}^{n-i} \sum_{j=i+k}^{n}\left(\begin{array}{c}
j+i-k-1 \\
2 i-1
\end{array}\right) \frac{A_{i j}^{(n)}}{2 D^{(n)}}\left(\Delta_{-k}^{[2 i]}+\Delta_{k}^{[2 i]}\right) x^{2 i} .
\end{aligned}
$$

For fixed $i, j$ the numbers

$$
\frac{A_{i j}^{(n)}}{2 D^{(n)}}=(-1)^{i+j} \frac{\left(\begin{array}{c}
2 n \\
n-j
\end{array}\right)}{\left(\begin{array}{c}
2 n \\
n
\end{array}\right)} \frac{1}{j^{2}} \sum \frac{1}{\sum^{2} \cdots r_{i-1}^{2}},
$$

where the summation indicated is that of products $i-1$ at a time of squared reciprocals of the first $n$ integers excepting $j$, are convergent functions of $n$, as $n$ is increased indefinitely. However, for fixed $i, k, i>1$, the terms of the sums with respect to $j$ in (2) are not bounded as $n$ and $j$ simultaneously increase. For this reason it is difficult to determine directly whether or not these sums converge.

* Presented to the Society, August 29, 1929; received by the editors in April, 1929.

$\dagger$ These Transactions, vol. 26 (1924), pp.113-123. 
If the values (1), $h=1, y_{0}=0$, are given for the infinite set of all positive and negative integers, the polynomials (2) constitute a sequence which may or may not be convergent. The polynomial (2) is identical with the partial sum of a Stirling series, and with the partial sum of a Gauss series, on condition that the terms of these series are grouped in pairs involving differences of order $2 i-1$ and $2 i$. The conditions of convergence of the series of Stirling and Gauss have been the subject of much recent literature.* It is known, for example, that whenever the polynomials (2) constitute a convergent sequence for some one non-integral value of $x$, this sequence is convergent for every value of $x$ to an integral function. $\dagger$

In this paper the sums with respect to $j$ in (2) will be transformed into sums the terms of which are independent of $n$, and are also of constant sign. The fact of convergence of the positive term sums which then express the coefficients of $\left(\Delta_{-k}{ }^{[m]}+\Delta_{k}[m]\right) x^{m}$, and of $\Delta_{0}^{[2 i]} x^{2 i}$ in this new form of (2) constitutes the main result of the paper.

The transformed form (39) of (2) is a triple series the partial sums of which when taken as indicated are identical with partial sums of the series of Gauss and Stirling (with the understanding that in these series terms are grouped in pairs as stated above). When this triple series is absolutely convergent its sum taken in the order $j, k, i$ is a power series representation of the integral. function which is then defined by the series of Gauss and Stirling. It seems appropriate to refer to this power series as the basic power series of interpolation.

2. Generating functions $P_{i k}^{(2 n-1)}, P_{i k}^{(2 n)}$. If the Lagrange polynomial (2) is an odd function it may be written (I, (3))

$$
\sum_{i=1}^{n} \sum_{j=1}^{n} j\left(y_{j}-y_{-j}\right) \frac{A_{i j}^{(n)}}{2 D^{(n)}} x^{2 i-1},
$$

and if by reference to (2) we place

$$
\begin{aligned}
y_{j}=-y_{-j} & =\left(\begin{array}{c}
j+i-k-1 \\
2 i-2
\end{array}\right), j=i+k-1, \cdots, n, \\
& =0, j<i+k-1,
\end{aligned}
$$

we obtain a polynomial for which the differences of order $2 i-1$ are all zero with the exception

$$
\Delta_{k}^{[2 i-1]}=\Delta_{-k}^{[2 i-1]}=1 .
$$

*Cf. Nörlund, Differenzenrechnung, Berlin, 1924, pp. 208-222, for references to this literature. $\dagger$ Nörlund, loc. cit., pp. 208-209. 
We shall call this polynomial a generating function, and shall denote it by the symbol $P_{i k}{ }^{(2 n-1)}$. With the exception noted its differences of order $2 i-1$ are all zero for all values of $n$.

Similarly, if the Lagrange polynomial (2) is an even function it may be written (I, (3))

$$
\sum_{i=1}^{n} \sum_{j=1}^{n}\left(y_{j}+y_{-j}\right) \frac{A_{i j}^{(n)}}{2 D^{(n)}} x^{2 i},
$$

and if by reference to (2) we place

$$
\begin{aligned}
y_{j}=y_{-j} & =\left(\begin{array}{c}
j+i-k-1 \\
2 i-1
\end{array}\right), j=i+k, \cdots, n, \\
& =0, j<i+k,
\end{aligned}
$$

we obtain a polynomial for which the differences of order $2 i$ are all zero with the exception

$$
\Delta_{k}^{[2 i]}=\Delta_{-k}^{[2 i]}=1, k>0 ;
$$

or

$$
\Delta_{0}^{[2 i]}=2, k=0 .
$$

We shall also call this polynomial a generating function, and shall denote it by the symbol $P_{i k}{ }^{(2 n)}$. With the exception noted its differences of order $2 i$ are all zero for all values of $n$.

It may be observed that the generating functions $P_{i k}{ }^{(2 n-1)}, P_{i k}{ }^{(2 n)}$ have properties with respect to differences of order $2 i-1,2 i$, respectively, wholly analogous to the properties with respect to values which characterize the polynomials which enter as coefficients into the formula of Lagrange in its classical form.

3. Central differences of the generating functions. Since we shall make essential use of the expansions of $P_{i k}^{(2 n-1)}$ and $P_{i k}{ }^{(2 n)}$ in the form of Stirling's series, which depend on central differences, we shall now compute these central differences from the given functional values, (5) and (8).

We shall first of all illustrate the general method by an example. Consider the generating function $P_{21}{ }^{(2 n-1)}$. Writing the values of this function, for $x=-n, \cdots, n$, and the successive differences of these values in horizontal rows, we have the following: 


$$
\begin{aligned}
& \cdots-\left(\begin{array}{l}
3 \\
2
\end{array}\right) \quad-\left(\begin{array}{l}
2 \\
2
\end{array}\right) \quad 0 \quad 000 \quad\left(\begin{array}{l}
2 \\
2
\end{array}\right) \quad\left(\begin{array}{l}
3 \\
2
\end{array}\right) \cdots \\
& \cdots\left(\begin{array}{l}
2 \\
1
\end{array}\right) \quad\left(\begin{array}{l}
1 \\
1
\end{array}\right) \quad 0 \quad 0 \quad\left(\begin{array}{l}
1 \\
1
\end{array}\right) \quad\left(\begin{array}{l}
2 \\
1
\end{array}\right) \ldots \\
& \begin{array}{llllllll}
\cdots & -1 & -1 & 0 & 1 & 1 & \ldots
\end{array} \\
& \begin{array}{llllll}
\cdots & 0 & 1 & 1 & 0 & \cdots
\end{array}
\end{aligned}
$$

Using the notation $(I,(38))$ for mean central differences of odd order which is commonly used in writing Stirling's series, we have

$$
\mu \Delta_{1}^{\prime \prime \prime}=1, \quad \mu \Delta_{1}^{\mathrm{V}}=-1, \quad \mu \Delta_{1}^{\mathrm{VII}}=2, \quad \mu \Delta_{1}^{\mathrm{IX}}=-5, \cdots .
$$

We may, of course, determine these mean central differences directly from the values of the function. Thus for $\mu \Delta_{1}$ VII we have

$$
\begin{aligned}
(11)-\left(\begin{array}{l}
7 \\
0
\end{array}\right)\left[-\left(\begin{array}{l}
3 \\
2
\end{array}\right)\right]+\left(\begin{array}{l}
7 \\
1
\end{array}\right)\left[-\left(\begin{array}{l}
2 \\
2
\end{array}\right)\right] & -\cdots \\
& +\left(\begin{array}{l}
7 \\
5
\end{array}\right)\left(\begin{array}{l}
2 \\
2
\end{array}\right)-\left(\begin{array}{l}
7 \\
6
\end{array}\right)\left(\begin{array}{l}
3 \\
2
\end{array}\right)+\left(\begin{array}{l}
7 \\
7
\end{array}\right)\left(\begin{array}{l}
4 \\
2
\end{array}\right) .
\end{aligned}
$$

Then by use of the identities*

$$
\begin{aligned}
& \sum_{s=0}^{m-q}(-1)^{\cdot}\left(\begin{array}{c}
m \\
q+s
\end{array}\right)\left(\begin{array}{c}
n+s \\
n
\end{array}\right)=\left(\begin{array}{c}
m-n-1 \\
m-q
\end{array}\right), \\
& \sum_{s=0}^{q}(-1)^{s}\left(\begin{array}{c}
m \\
q-s
\end{array}\right)\left(\begin{array}{c}
n+s \\
n
\end{array}\right)=\left(\begin{array}{c}
m-n-1 \\
q
\end{array}\right), \\
& \left(\begin{array}{c}
2 n \\
n-k
\end{array}\right)-\left(\begin{array}{c}
2 n \\
n-k-1
\end{array}\right)=\frac{2 k+1}{2 n+1}\left(\begin{array}{c}
2 n+1 \\
n-k
\end{array}\right),
\end{aligned}
$$

we obtain

$$
\mu \Delta_{1}^{\mathrm{VII}}=\left(\begin{array}{l}
4 \\
2
\end{array}\right)-\left(\begin{array}{l}
4 \\
1
\end{array}\right)=\frac{1}{5}\left(\begin{array}{l}
5 \\
2
\end{array}\right) .
$$

The identities (12) suffice for the determination of the mean central differences and central differences for all the generating functions $P_{i k}{ }^{(2 n-1)}$, $P_{i k}{ }^{(2 n)}$, the manner of application being precisely that of the foregoing example. It will suffice, therefore, if we tabulate results, as follows:

* Netto, Combinatorik, p. 255. 
Central differences of $P_{i k}{ }^{(2 n-1)}$

(14)

$$
\begin{aligned}
& i=1: \quad \mu \Delta_{1}^{\prime} \quad \mu \Delta_{1}^{\prime \prime \prime} \quad \mu \Delta_{1}^{\mathrm{V}} \quad \mu \Delta_{1}^{\text {VII }} \\
& P_{11}^{(2 n-1)} \frac{1}{1}\left(\begin{array}{l}
1 \\
0
\end{array}\right)-\frac{1}{3}\left(\begin{array}{l}
3 \\
1
\end{array}\right) \quad \frac{1}{5}\left(\begin{array}{l}
5 \\
2
\end{array}\right)-\frac{1}{7}\left(\begin{array}{l}
7 \\
3
\end{array}\right) \ldots \\
& P_{12}^{(2 n-1)} \quad \frac{3}{3}\left(\begin{array}{l}
3 \\
0
\end{array}\right) \quad-\frac{3}{5}\left(\begin{array}{l}
5 \\
1
\end{array}\right) \quad \frac{3}{7}\left(\begin{array}{l}
7 \\
2
\end{array}\right) \cdots \\
& P_{13}^{(2 n-1)} \\
& \frac{5}{5}\left(\begin{array}{l}
5 \\
0
\end{array}\right)-\frac{5}{7}\left(\begin{array}{l}
7 \\
1
\end{array}\right) \cdots \\
& i=2 \text { : } \\
& \mu \Delta_{1}^{\prime \prime \prime} \\
& \mu \Delta_{1}^{\mathrm{v}} \quad \mu \Delta_{1}^{\mathrm{VII}} \\
& P_{21}^{(2 n-1)} \\
& \frac{1}{1}\left(\begin{array}{l}
1 \\
0
\end{array}\right) \quad-\frac{1}{3}\left(\begin{array}{l}
3 \\
1
\end{array}\right) \quad \frac{1}{5}\left(\begin{array}{l}
5 \\
2
\end{array}\right) \cdots \\
& P_{22}^{(2 n-1)} \\
& \frac{3}{3}\left(\begin{array}{l}
3 \\
0
\end{array}\right) \quad-\frac{3}{5}\left(\begin{array}{l}
5 \\
1
\end{array}\right) \cdots \\
& P_{23}^{(2 n-1)} \\
& \frac{5}{5}\left(\begin{array}{l}
5 \\
0
\end{array}\right) \ldots \\
& i=3 \text { : } \\
& \mu \Delta_{1}^{\mathrm{V}} \quad \mu \Delta_{1}^{\mathrm{VII}} \\
& P_{31}^{(2 n-1)} \\
& \frac{1}{1}\left(\begin{array}{l}
1 \\
0
\end{array}\right)-\frac{1}{3}\left(\begin{array}{l}
3 \\
1
\end{array}\right) \cdots \\
& P_{32}^{(2 n-1)} \\
& \frac{3}{3}\left(\begin{array}{l}
3 \\
0
\end{array}\right) \cdots
\end{aligned}
$$

Central differences of $P_{i k}^{(2 n)}$

$$
\begin{aligned}
& \text { (15) } i=1: \quad \Delta_{0}^{\prime \prime} \\
& \Delta_{0}^{\mathrm{IV}} \\
& \Delta_{0}^{\mathrm{VI}} \\
& \begin{array}{l}
\text { viII } \\
\Delta_{0}
\end{array} \\
& P_{10}^{(2 n)} \\
& 2\left(\begin{array}{l}
0 \\
0
\end{array}\right) \\
& -2\left(\begin{array}{l}
2 \\
1
\end{array}\right) \\
& 2\left(\begin{array}{l}
4 \\
2
\end{array}\right) \\
& -2\left(\begin{array}{l}
6 \\
3
\end{array}\right) \ldots \\
& P_{11}^{(2 n)} \\
& 2\left(\begin{array}{l}
2 \\
0
\end{array}\right) \\
& -2\left(\begin{array}{l}
4 \\
1
\end{array}\right) \\
& 2\left(\begin{array}{l}
6 \\
2
\end{array}\right) \ldots \\
& P_{12}^{(2 n)} \\
& 2\left(\begin{array}{l}
4 \\
0
\end{array}\right) \quad-2\left(\begin{array}{l}
6 \\
1
\end{array}\right) \cdots
\end{aligned}
$$




$$
\begin{aligned}
& i=2 \text { : } \\
& \Delta_{0}^{\text {IV }} \\
& \Delta_{0}^{\mathbf{V I}} \\
& \Delta_{0}^{\text {vIII }} \\
& P_{20}^{(2 n)} \\
& 2\left(\begin{array}{l}
0 \\
0
\end{array}\right) \quad-2\left(\begin{array}{l}
2 \\
1
\end{array}\right) \quad 2\left(\begin{array}{l}
4 \\
2
\end{array}\right) \ldots \\
& P_{21}^{(2 n)} \\
& 2\left(\begin{array}{l}
2 \\
0
\end{array}\right) \quad-2\left(\begin{array}{l}
4 \\
1
\end{array}\right) \ldots \\
& P_{22}^{(2 n)} \\
& 2\left(\begin{array}{l}
4 \\
0
\end{array}\right) \cdots \\
& i=3 \text { : } \\
& \Delta_{0}^{\text {VI }} \quad \Delta_{0}^{\text {VIII }} \\
& P_{30}^{(2 n)} \\
& 2\left(\begin{array}{l}
0 \\
0
\end{array}\right) \\
& -2\left(\begin{array}{l}
2 \\
1
\end{array}\right) \cdots \\
& P_{81}^{(2 n)} \\
& 2\left(\begin{array}{l}
2 \\
0
\end{array}\right) \cdots
\end{aligned}
$$

4. The Stirling series for $P_{i k}^{(2 n-1)}, P_{i k}^{(2 n)}$. Corresponding to the mean differences (14) we have the following Stirling series expansions:

$$
\begin{aligned}
P_{11}^{(2 n-1)}=\frac{1}{1}\left(\begin{array}{l}
1 \\
0
\end{array}\right) \frac{1}{1 !} x-\frac{1}{3}\left(\begin{array}{l}
3 \\
1
\end{array}\right) \frac{1}{3 !} x\left(x^{2}-1\right) & +\frac{1}{5}\left(\begin{array}{l}
5 \\
2
\end{array}\right) \frac{1}{5 !} x\left(x^{2}-1\right)\left(x^{2}-2^{2}\right)-\cdots, \\
P_{12}^{(2 n-1)}=\frac{3}{3}\left(\begin{array}{l}
3 \\
0
\end{array}\right) \frac{1}{3 !} x\left(x^{2}-1\right) & -\frac{3}{5}\left(\begin{array}{l}
5 \\
1
\end{array}\right) \frac{1}{5 !} x\left(x^{2}-1\right)\left(x^{2}-2^{2}\right)+\cdots, \\
P_{13}^{(2 n-1)}= & \frac{5}{5}\left(\begin{array}{l}
5 \\
0
\end{array}\right) \frac{1}{5 !} x\left(x^{2}-1\right)\left(x^{2}-2^{2}\right)-\cdots, \\
P_{21}^{(2 n-1)}=\frac{1}{1}\left(\begin{array}{l}
1 \\
0
\end{array}\right) \frac{1}{3 !} x\left(x^{2}-1\right)- & \frac{1}{3}\left(\begin{array}{l}
3 \\
1
\end{array}\right) \frac{1}{5 !} x\left(x^{2}-1\right)\left(x^{2}-2^{2}\right)+\cdots, \\
P_{22}^{(2 n-1)}= & \frac{3}{3}\left(\begin{array}{l}
3 \\
0
\end{array}\right) \frac{1}{5 !} x\left(x^{2}-1\right)\left(x^{2}-2^{2}\right)-\cdots,
\end{aligned}
$$


These expansions are then reducible to the following form:

$$
\begin{aligned}
& P_{11}^{(2 n-1)}=\frac{1}{1}\left(\begin{array}{l}
1 \\
0
\end{array}\right) \frac{2}{\left(\begin{array}{l}
2 \\
1
\end{array}\right)} \frac{x}{1^{2}}-\frac{1}{3}\left(\begin{array}{l}
3 \\
1
\end{array}\right) \frac{4}{\left(\begin{array}{l}
4 \\
2
\end{array}\right)} \frac{x\left(x^{2}-1\right)}{1^{2} 2^{2}} \\
& +\frac{1}{5}\left(\begin{array}{l}
5 \\
2
\end{array}\right) \frac{6}{\left(\begin{array}{l}
6 \\
3
\end{array}\right)} \frac{x\left(x^{2}-1\right)\left(x^{2}-2^{2}\right)}{1^{2} 2^{2} 3^{2}}+\cdots, \\
& P_{12}^{(2 n-1)}=\frac{3}{3}\left(\begin{array}{l}
3 \\
0
\end{array}\right) \frac{4}{\left(\begin{array}{l}
4 \\
2
\end{array}\right)} \frac{x\left(x^{2}-1\right)}{1^{2} 2^{2}} \\
& -\frac{3}{5}\left(\begin{array}{l}
5 \\
1
\end{array}\right) \frac{6}{\left(\begin{array}{l}
6 \\
3
\end{array}\right)} \frac{x\left(x^{2}-1\right)\left(x^{2}-2^{2}\right)}{1^{2} 2^{2} 3^{2}}+\cdots, \\
& P_{13}^{(2 n-1)}=\frac{5}{5}\left(\begin{array}{l}
5 \\
0
\end{array}\right) \frac{6}{\left(\begin{array}{l}
6 \\
3
\end{array}\right)} \frac{x\left(x^{2}-1\right)\left(x^{2}-2^{2}\right)}{1^{2} 2^{2} 3^{2}}-\cdots, \\
& P_{21}^{(2 n-1)}=\frac{1}{1}\left(\begin{array}{l}
1 \\
0
\end{array}\right) \frac{4}{\left(\begin{array}{l}
4 \\
2
\end{array}\right)} \frac{x\left(x^{2}-1\right)}{1^{2} 2^{2}} \\
& -\frac{1}{3}\left(\begin{array}{l}
3 \\
1
\end{array}\right) \frac{6}{\left(\begin{array}{l}
6 \\
3
\end{array}\right)} \frac{x\left(x^{2}-1\right)\left(x^{2}-2^{2}\right)}{1^{2} 2^{2} 3^{2}}+\cdots, \\
& P_{22}^{(2 n-1)}=\frac{3}{3}\left(\begin{array}{l}
3 \\
0
\end{array}\right) \frac{6}{\left(\begin{array}{l}
6 \\
3
\end{array}\right)} \frac{x\left(x^{2}-1\right)\left(x^{2}-2^{2}\right)}{1^{2} 2^{2} 3^{2}}-\cdots,
\end{aligned}
$$

In like manner we have Stirling series expansions corresponding to the central differences (15), as follows:

(18) $P_{10}^{(2 n)}=2\left(\begin{array}{l}0 \\ 0\end{array}\right) \frac{1}{2 !} x^{2}-2\left(\begin{array}{l}2 \\ 1\end{array}\right) \frac{1}{4 !} x^{2}\left(x^{2}-1\right)$

$$
+2\left(\begin{array}{l}
4 \\
2
\end{array}\right) \frac{1}{6 !} x^{2}\left(x^{2}-1\right)\left(x^{2}-2^{2}\right)-\cdots,
$$




$$
\begin{aligned}
& P_{11}^{(2 n)}=2\left(\begin{array}{l}
2 \\
0
\end{array}\right) \frac{1}{4 !} x^{2}\left(x^{2}-1\right)-2\left(\begin{array}{l}
4 \\
1
\end{array}\right) \frac{1}{6 !} x^{2}\left(x^{2}-1\right)\left(x^{2}-2^{2}\right)+\cdots, \\
& P_{12}^{(2 n)}=2\left(\begin{array}{l}
4 \\
0
\end{array}\right) \frac{1}{6 !} x^{2}\left(x^{2}-1\right)\left(x^{2}-2^{2}\right)-\cdots, \\
& P_{20}^{(2 n)}=2\left(\begin{array}{l}
0 \\
0
\end{array}\right) \frac{1}{4 !} x^{2}\left(x^{2}-1\right)-2\left(\begin{array}{l}
2 \\
1
\end{array}\right) \frac{1}{6 !} x^{2}\left(x^{2}-1\right)\left(x^{2}-2^{2}\right)+\cdots, \\
& P_{21}^{(2 n)}=2\left(\begin{array}{l}
2 \\
0
\end{array}\right) \frac{1}{6 !} x^{2}\left(x^{2}-1\right)\left(x^{2}-2^{2}\right)-\cdots,
\end{aligned}
$$

The expansions (18) are reducible as follows:

(19)

$$
\begin{aligned}
& P_{10}^{(2 n)}=\left(\begin{array}{l}
0 \\
0
\end{array}\right) \frac{2}{\left(\begin{array}{l}
2 \\
1
\end{array}\right)} \frac{x^{2}}{1^{2}}-\left(\begin{array}{l}
2 \\
1
\end{array}\right) \frac{2}{\left(\begin{array}{l}
4 \\
2
\end{array}\right)} \frac{x^{2}\left(x^{2}-1\right)}{1^{2} 2^{2}} \\
& +\left(\begin{array}{l}
4 \\
2
\end{array}\right) \frac{2}{\left(\begin{array}{l}
6 \\
3
\end{array}\right)} \frac{x^{2}\left(x^{2}-1\right)\left(x^{2}-2^{2}\right)}{1^{2} 2^{2} 3^{2}}-\cdots, \\
& P_{11}^{(2 n)}=\left(\begin{array}{l}
2 \\
01
\end{array}\right) \frac{2}{\left(\begin{array}{l}
4 \\
2
\end{array}\right)} \frac{x^{2}\left(x^{2}-1\right)}{1^{2} 2^{2}}-\left(\begin{array}{l}
4 \\
1
\end{array}\right) \frac{2}{\left(\begin{array}{l}
6 \\
3
\end{array}\right)} \frac{x^{2}\left(x^{2}-1\right)\left(x^{2}-2^{2}\right)}{1^{2} 2^{2} 3^{2}}+\cdots, \\
& P_{12}^{(2 n)}=\left(\begin{array}{l}
4 \\
0
\end{array}\right) \frac{2}{\left(\begin{array}{l}
6 \\
3
\end{array}\right)} \frac{x^{2}\left(x^{2}-1\right)\left(x^{2}-2^{2}\right)}{1^{2} 2^{2} 3^{2}}-\cdots, \\
& P_{20}^{(2 n)}=\left(\begin{array}{l}
0 \\
0
\end{array}\right) \frac{2}{\left(\begin{array}{l}
4 \\
2
\end{array}\right)} \frac{x^{2}\left(x^{2}-1\right)}{1^{2} 2^{2}}-\left(\begin{array}{l}
2 \\
1
\end{array}\right) \frac{2}{\left(\begin{array}{l}
6 \\
3
\end{array}\right)} \frac{x^{2}\left(x^{2}-1\right)\left(x^{2}-2^{2}\right)}{1^{2} 2^{2} 3^{2}}+\cdots, \\
& P_{21}^{(2 n)}=\left(\begin{array}{l}
2 \\
0
\end{array}\right) \frac{2}{\left(\begin{array}{l}
6 \\
3
\end{array}\right)} \frac{x^{2}\left(x^{2}-1\right)\left(x^{2}-2^{2}\right)}{1^{2} 2^{2} 3^{2}}-\cdots,
\end{aligned}
$$


From the definition of $P_{i k}^{(2 n-1)}$ and by reference to (2), it follows that, for fixed $i, k$, the coefficient of $x^{2 i-1}$ in the expansion (17) of $P_{i k}{ }^{(2 n-1)}$ is equal to

$$
2 \sum_{j=i+k-1}^{n} j\left(\begin{array}{c}
j+i-k-1 \\
2 i-2
\end{array}\right) \frac{A_{i j}^{(n)}}{2 D^{(n)}}
$$

Similarly, from the definition of $P_{i k}^{(2 n)}$ and by reference to (2), it follows that, for fixed $i, k$, the coefficient of $x^{2 i}$ in the expansion (19) of $P_{i k}{ }^{(2 n)}$ is equal to

$$
2 \sum_{j=i+k}^{n}\left(\begin{array}{c}
j+i-k-1 \\
2 i-1
\end{array}\right) \frac{A_{i j}^{(n)}}{2 D^{(n)}} .
$$

For fixed $i, k, i>1$, the terms of (20) and (21) are not bounded as $j$ and $n$ simultaneously increase, but the equivalent expressions derived from (17) and (19) consist of terms of constant sign and independent of $n$. This we shall consider in the next section.

5. The coefficients of $x^{2 i-1}, x^{2 i}$ in $P_{i k}^{(2 n-1)}, P_{i k}^{(2 n)}$. In this section we shall write down from (17) and (19) the coefficients which are equal to (20) and (21) respectively. We shall consider first the odd powers $x^{2 i-1}$, then the even powers $x^{2 i}$. For any odd power we have $k=1,2, \cdots$, and for any even power we have $k=0,1, \cdots$. The tabulation, arranged according to values of $i$ and $k$, is derived directly in an evident manner from the expansions 17) and (19).

$$
\left.P_{1 k}^{(2 n-1)} \text { (Coefficient of } x\right) \text { : }
$$

(22) $k=1 \quad\left\{\frac{1}{1}\left(\begin{array}{l}1 \\ 0\end{array}\right) \frac{2}{\left(\begin{array}{l}2 \\ 1\end{array}\right)} \frac{1}{1^{2}}+\frac{1}{3}\left(\begin{array}{l}3 \\ 1\end{array}\right) \frac{4}{\left(\begin{array}{l}4 \\ 2\end{array}\right)} \frac{1}{2^{2}}\right.$

$$
\begin{gathered}
\left.+\frac{1}{5}\left(\begin{array}{l}
5 \\
2
\end{array}\right) \frac{6}{\left(\begin{array}{l}
6 \\
3
\end{array}\right)} \frac{1}{3^{2}}+\cdots\right\}, \\
k=2 \quad-\left\{\frac{3}{3}\left(\begin{array}{l}
3 \\
0
\end{array}\right) \frac{4}{\left(\begin{array}{l}
4 \\
2
\end{array}\right)} \frac{1}{2^{2}}+\frac{3}{5}\left(\begin{array}{l}
5 \\
1
\end{array}\right) \frac{6}{\left(\begin{array}{l}
6 \\
3
\end{array}\right)} \frac{1}{3^{2}}+\cdots\right\},
\end{gathered}
$$


$P_{2 k}^{(2 n-1)}\left(\right.$ Coefficient of $\left.x^{3}\right)$ :

(23) $k=1\left\{\frac{1}{1}\left(\begin{array}{l}1 \\ 0\end{array}\right) \frac{4}{\left(\begin{array}{l}4 \\ 2\end{array}\right)} \frac{1}{2^{2}} \sum_{1}^{1} \frac{1^{*}}{r_{1}^{2}}\right.$

$$
\begin{aligned}
& \left.+\frac{1}{3}\left(\begin{array}{l}
3 \\
1
\end{array}\right) \frac{6}{\left(\begin{array}{l}
6 \\
3
\end{array}\right)} \frac{1}{3^{2}} \sum_{1}^{2} \frac{1}{r_{1}^{2}}+\cdots\right\}, \\
& \quad-\left\{\frac{3}{3}\left(\begin{array}{l}
3 \\
0
\end{array}\right) \frac{6}{\left(\begin{array}{l}
6 \\
3
\end{array}\right)} \frac{1}{3^{2}} \sum_{1}^{2} \frac{1}{r_{1}^{2}}+\cdots\right\},
\end{aligned}
$$

$P_{3 k}^{(2 n-1)}$ (Coefficient of $\left.x^{5}\right)$ :

(24) $k=1 \quad\left\{\frac{1}{1}\left(\begin{array}{l}1 \\ 0\end{array}\right) \frac{6}{\left(\begin{array}{l}6 \\ 3\end{array}\right)} \frac{1}{3^{2}} \sum_{1}^{2} \frac{1^{*}}{r_{1}^{2} r_{2}^{2}}\right.$

$$
\begin{aligned}
+\frac{1}{3}\left(\begin{array}{l}
3 \\
1
\end{array}\right) & \left.\frac{8}{\left(\begin{array}{l}
8 \\
4
\end{array}\right)} \frac{1}{4^{2}} \sum_{1}^{3} \frac{1}{r_{1}^{2} r_{2}^{2}}+\cdots\right\}, \\
& -\left\{\frac{3}{3}\left(\begin{array}{l}
3 \\
0
\end{array}\right) \frac{8}{\left(\begin{array}{l}
8 \\
4
\end{array}\right)} \frac{1}{4^{2}} \sum_{1}^{3} \frac{1}{r_{1}^{2} r_{2}^{2}}+\cdots\right\},
\end{aligned}
$$

$P_{4 k}^{(2 n-1)}\left(\right.$ Coefficient of $\left.x^{7}\right)$ :

(25) $k=1 \quad\left\{\frac{1}{1}\left(\begin{array}{l}1 \\ 0\end{array}\right) \frac{8}{\left(\begin{array}{l}8 \\ 4\end{array}\right)} \frac{1}{4^{2}} \sum_{1}^{3} \frac{1^{*}}{r_{1}^{2} r_{2}{ }^{2} r_{3}{ }^{2}}\right.$

$$
\begin{aligned}
& \left.+\frac{1}{3}\left(\begin{array}{l}
3 \\
1
\end{array}\right) \frac{10}{\left(\begin{array}{c}
10 \\
5
\end{array}\right)} \frac{1}{5^{2}} \sum_{1}^{4} \frac{1}{r_{1}^{2} r_{2}{ }^{2} r_{3}^{2}}+\cdots\right\}, \\
k=2 & -\left\{\frac{3}{3}\left(\begin{array}{l}
3 \\
0
\end{array}\right) \frac{10}{\left(\begin{array}{c}
10 \\
5
\end{array}\right)} \frac{1}{5^{2}} \sum_{1}^{4} \frac{1}{r_{1}^{2} r_{2}^{2} r_{3}^{2}}+\cdots\right\},
\end{aligned}
$$

* Sums reducing to a single term are included to preserve symmetry. 
$P_{1 k}^{(2 n)}$ (Coefficient of $\left.x^{2}\right)$ :

(26) $k=0 \quad\left\{\left(\begin{array}{l}0 \\ 0\end{array}\right) \frac{2}{\left(\begin{array}{l}2 \\ 1\end{array}\right)} \frac{1}{1^{2}}\right.$

$$
\begin{array}{ll} 
& \left.+\left(\begin{array}{l}
2 \\
1
\end{array}\right) \frac{2}{\left(\begin{array}{l}
4 \\
2
\end{array}\right)} \frac{1}{2^{2}}+\left(\begin{array}{l}
4 \\
2
\end{array}\right) \frac{2}{\left(\begin{array}{l}
6 \\
3
\end{array}\right)} \frac{1}{3^{2}}+\cdots\right\}, \\
k=1 & -\left\{\left(\begin{array}{l}
2 \\
0
\end{array}\right) \frac{2}{\left(\begin{array}{l}
4 \\
2
\end{array}\right)} \frac{1}{2^{2}}+\left(\begin{array}{l}
4 \\
1
\end{array}\right) \frac{2}{\left(\begin{array}{l}
6 \\
3
\end{array}\right)} \frac{1}{3^{2}}+\cdots\right\},
\end{array}
$$

$P_{2 k}^{(2 n)}$ (Coefficient of $\left.x^{4}\right)$ :

(27) $k=0 \quad\left\{\left(\begin{array}{l}0 \\ 0\end{array}\right) \frac{2}{\left(\begin{array}{l}4 \\ 2\end{array}\right)} \frac{1}{2^{2}} \sum_{1}^{1} \frac{1}{r_{1}{ }^{2}}\right.$

$$
\begin{aligned}
& \left.+\left(\begin{array}{l}
2 \\
1
\end{array}\right) \frac{2}{\left(\begin{array}{l}
6 \\
3
\end{array}\right)} \frac{1}{3^{2}} \sum_{1}^{2} \frac{1}{r_{1}^{2}}+\cdots\right\}, \\
& \quad-\left\{\left(\begin{array}{l}
2 \\
0
\end{array}\right) \frac{2}{\left(\begin{array}{l}
6 \\
3
\end{array}\right)} \frac{1}{3^{2}} \sum_{1}^{2} \frac{1}{r_{1}^{2}}+\cdots\right\},
\end{aligned}
$$

$P_{3 k}^{(2 n)}\left(\right.$ Coefficient of $\left.x^{6}\right)$ :

(28) $k=0 \quad\left\{\left(\begin{array}{l}0 \\ 0\end{array}\right) \frac{2}{\left(\begin{array}{l}6 \\ 3\end{array}\right)} \frac{1}{3^{2}} \sum_{1}^{2} \frac{1}{r_{1}^{2} r_{2}^{2}}\right.$

$$
\left.+\left(\begin{array}{l}
2 \\
1
\end{array}\right) \frac{2}{\left(\begin{array}{l}
8 \\
4
\end{array}\right)} \frac{1}{4^{2}} \sum_{1}^{3} \frac{1}{r_{1}^{2} r_{2}^{2}}+\cdots\right\},
$$

$k=1$

$$
-\left\{\left(\begin{array}{l}
2 \\
0
\end{array}\right) \frac{2}{\left(\begin{array}{l}
8 \\
4
\end{array}\right)} \frac{1}{4^{2}} \sum_{1}^{8} \frac{1}{r_{1}^{2} r_{2}^{2}}+\cdots\right\},
$$


$P_{4 k}^{(2 n)}\left(\right.$ Coefficient of $\left.x^{8}\right)$ :

(29) $k=0 \quad\left\{\left(\begin{array}{l}0 \\ 0\end{array}\right) \frac{2}{\left(\begin{array}{l}8 \\ 4\end{array}\right)} \frac{1}{4^{2}} \sum_{1}^{3} \frac{1}{r_{1}^{2} r_{2}^{2} r_{3}^{2}}\right.$

$$
\begin{aligned}
& \left.+\left(\begin{array}{l}
2 \\
1
\end{array}\right) \frac{2}{\left(\begin{array}{c}
10 \\
5
\end{array}\right)} \frac{1}{5^{2}} \sum_{1}^{4} \frac{1}{r_{1}^{2} r_{2}^{2} r_{3}^{2}}+\cdots\right\}, \\
& -\left\{\left(\begin{array}{l}
2 \\
0
\end{array}\right) \frac{2}{\left(\begin{array}{c}
10 \\
5
\end{array}\right)} \frac{1}{5^{2}} \sum_{1}^{4} \frac{1}{r_{1}^{2} r_{2}^{2} r_{3}^{2}}+\cdots\right\},
\end{aligned}
$$$$
k=1
$$

6. Identities. If we observe that

$$
\left|\frac{A_{i j}^{(j)}}{2 D^{(j)}}\right|=\frac{1}{\left(\begin{array}{c}
2 j \\
j
\end{array}\right)} \frac{1}{j^{2}} \sum_{1}^{j-1} \frac{1}{r_{1}^{2} \cdots r_{i-1}^{2}},
$$

we may express the equality between (20), (21) and the corresponding sums appearing in (22) to (29) inclusive, as follows:

$$
\begin{aligned}
& \quad \sum_{j=i+k-1}^{n}\left(\begin{array}{c}
j+i-k-1 \\
2 i-2
\end{array}\right) j \frac{A_{i j}^{(n)}}{2 D^{(n)}} \\
& \quad=(-1)^{k+1} \sum_{j=i+i-1}^{n} \frac{2 k-1}{2 j-2 i+1}\left(\begin{array}{c}
2 j-2 i+1 \\
j-i-k+1
\end{array}\right) j\left|\frac{A_{i j}^{(j)}}{2 D^{(j)}}\right|,
\end{aligned}
$$

$$
\begin{aligned}
& \sum_{j=i+k}^{n}\left(\begin{array}{c}
j+i-k-1 \\
2 i-1
\end{array}\right) \frac{A_{i j}^{(n)}}{2 D^{(n)}} \\
& \quad=(-1)^{k} \sum_{j=i+k}^{n}\left(\begin{array}{c}
2 j-2 i \\
j-i-k
\end{array}\right)\left|\frac{A_{i j}^{(j)}}{2 D^{(j)}}\right|
\end{aligned}
$$

In (31) $k$ has the values $1,2, \cdots$, and in (32) the values $0,1,2, \cdots$. The significance of these identities lies first of all in the fact that the terms on the right are not functions of $\boldsymbol{n}$ and moreover are all of one sign. Of greater significance, however, is the fact that the positive term series on the right of these identities are convergent as $n$ is increased indefinitely. This we shall now prove. 
It may be seen at once that

$$
\sum_{j=1}^{\infty}\left(\begin{array}{c}
2 j \\
j
\end{array}\right)\left|\frac{A_{i j}^{(j)}}{2 D^{(i)}}\right|=\sum_{j=1}^{\infty} \frac{1}{j^{2}} \sum_{1}^{j-1} \frac{1}{r_{1}^{2} \cdots r_{i-1}^{2}}
$$

is a particular arrangement of the products $i$ at a time of the squared reciprocals of all positive integers. This sum is well known through its connection with

$$
\frac{\sinh \pi x}{\pi x}=\prod_{1}^{\infty}\left(1+\frac{x^{2}}{n^{2}}\right)
$$

Therefore (33) is convergent, as follows:

$$
\sum_{j=1}^{\infty}\left(\begin{array}{c}
2 j \\
j
\end{array}\right)\left|\frac{A_{i j}^{(j)}}{2 D^{(j)}}\right|=\frac{\pi^{2 i}}{(2 i+1) !} .
$$

If we now place $j=i+k+s, s=0,1,2, \cdots$, we obtain for the ratio by which we may compare (31) with (33),

(36) $\frac{(2 k+2 s+1) \cdots(2 k+s+1)}{(2 k+2 s+2 i) \cdots(2 k+s+2 i)}$

$$
\frac{(s+k+i) \cdots(s+2)}{(s+2 k+2 i-1) \cdots(s+k+i+1)} \frac{s+k+i}{2 s+2 k+1}(2 k-1),
$$

which is the value in fractional form of the ratio

$$
\frac{2 k-1}{2 j-2 i+1} \frac{j\left(\begin{array}{c}
2 j-2 i+1 \\
j-i-k+1
\end{array}\right)}{\left(\begin{array}{c}
2 j \\
j
\end{array}\right)}
$$

If in (36) we place $s=i+r, r=0,1,2, \cdots$, we see that for $j \geqq 2 i+k$ the ratio (37) is less than $2 k-1$. Thus (31) is convergent by comparison with (33).

If we compare (32) with (33), we see directly that for $j \geqq i+k$

$$
\frac{\left(\begin{array}{c}
2 j-2 i \\
j-i-k
\end{array}\right)}{\left(\begin{array}{c}
2 j \\
j
\end{array}\right)}<1 .
$$

Thus the convergence of (32) is established. 
7. The power series of interpolation. The identities (31), (32) serve to effect the transformation of (2) into the following form:

$\sum_{i=1}^{n} \sum_{k=1}^{n-i+1}(-1)^{k+1} \sum_{j=i+k-1}^{n} \frac{2 k-1}{2 j-2 i+1}$

$$
\left(\begin{array}{c}
2 j-2 i+1 \\
j-i-k+1
\end{array}\right) j\left|\frac{A_{i j}^{(j)}}{2 D^{(j)}}\right|\left(\Delta_{-k}^{[2 i-1]}+\Delta_{k}^{[2 i-1]}\right) x^{2 i-1}
$$

$+\sum_{i=1}^{n} \sum_{j=i}^{n}\left(\begin{array}{c}2 j-2 i \\ j-i\end{array}\right)\left|\frac{A_{i j}^{(j)}}{2 D^{(j)}}\right| \Delta_{0}^{[2 i]} x^{2 i}$

$+\sum_{i=1}^{n} \sum_{k=1}^{n-i}(-1)^{k} \sum_{j=i+k}^{n}\left(\begin{array}{c}2 j-2 i \\ j-i-k\end{array}\right)\left|\frac{A_{i j}^{(j)}}{2 D^{(j)}}\right|\left(\Delta_{-k}^{[2 i]}+\Delta_{k}^{[2 i]}\right) x^{2 i}$.

It has been proved in the preceding section that the sums with respect to $j$ in (39) are convergent positive-term series. This theorem, together with the explicit form of (39), constitutes the main objective of this paper.

If the triple series (39) is absolutely convergent for a particular set of values (1), $h=1, y_{0}=0$, then the interpolation series of Stirling and Gauss are convergent (for this means only that the limit of the finite sum (39) exists as $n \rightarrow \infty)$, and if (39) is summed in the order $j, k, i$ it becomes the power series representation of the limit. It is possibly true conversely that (39) is absolutely convergent whenever the Gauss and Stirling series, with paired terms, are convergent. The series (39) has therefore a fundamental place in the theory of Gauss and Stirling series.

Massachusetrs Institute of Technology, Cambridge, Mass. 